\title{
Purple urine bag syndrome is a rare manifestation of bacteriuria on bladder catheter: case report
}

\author{
Othmane Mohib* (), Thomas Roland, Margot Fontaine, France Laurent and Camelia Rossi
}

\begin{abstract}
Background: Purple urine bag syndrome (PUBS) is a complication of bacterial colonisation of bladder catheters in which urine turns purple in the tubing, as well as in the catheter bag. This rare phenomenon can be very worrisome and stressful for the patients and their families, as well as for the healthcare team taking care of them. Recognising this complication is essential in order to avoid misdiagnosis and erroneous treatment. We report a case of PUBS in a 71-year-old female patient.

Case presentation: A 71-year-old woman with previous medical history of schizophrenia was admitted to the emergency department for anorexia and suspicion of psychotic decompensation. Acute urine retention and rectal faecal impaction were clinically suspected and confirmed by bladder ultrasound and rectal examination, respectively. The patient underwent bladder catheterisation as well as a rectal enema. The day after her admission, our medical team was approached by the nurse in charge of the patient because of purple urine in her catheter bag and tubing. The diagnosis of PUBS was made with the help of the Oxford urine chart. A dipstick urinalysis revealed alkaline urine $(\mathrm{pH}=8)$, and the urine culture was subsequently positive for Proteus mirabilis, which is sensitive to quinolones, betalactams and nitrofurantoin. The bladder catheter was changed. The patient received empiric antibiotic therapy with Levofloxacin $500 \mathrm{mg}$ once daily. After obtaining the antibiogram, the targeted antibiotic therapy was adapted with oral Cefuroxime $500 \mathrm{mg}$ three times a day for a total duration of seven days of antibiotic therapy. There was no recurrence of purple urine.

Conclusion: PUBS is a rare complication of bacteriuria, which induces a purple colouration of the tubing as well as the catheter bag. It is a simple spot diagnosis, as there is no other known cause of purple urine. This is why we believe that the Oxford urine chart represents a very interesting and easily accessible tool to help clinicians to investigate any abnormal urine colour.
\end{abstract}

Keywords: Purple, Urine, Bag, Infection, Bladder, Catheterisation, Case, Report

\section{Background}

Purple urine bag syndrome (PUBS) is a complication of bacterial colonisation of bladder catheters in which urine turns purple in the tubing as well as in the catheter bag.
*Correspondence: Othmane.Mohib@ulb.be

Department of Infectious Diseases, Centre Hospitalier Universitaire et Psychiatrique de Mons-Borinage, Mons, Belgium
This rare phenomenon can be very worrisome and stressful for patients and their families, as well as for the healthcare team taking care of them.

This is a simple spot diagnosis [1]. Recognising this rare complication is essential in order to avoid misdiagnosis and erroneous treatment.

We report the case of a 71-year-old patient who presented with PUBS following a bladder catheterisation during her stay in our internal medicine department. 


\section{Case presentation}

A 71-year-old woman with schizophrenia was referred to the emergency department by her attending physician on suspicion of psychotic decompensation and after having had anorexia for a month. The patient's medical history could not be taken because she presented with incoherent speech. On clinical examination, cachexia, signs of extracellular dehydration and hypogastric abdominal pain with dull percussion were observed. A bladder ultrasound revealed acute retention of urine leading to indwelling bladder catheterisation. A rectal examination was then performed and suggested a rectal faecal impaction, which could be removed by a rectal enema. A routine blood test revealed only a slight inflammatory syndrome as an abnormality.

The day after the patient's admission, our medical team was approached by the nurse in charge of the patient because of purple urine in her catheter bag and tubing (Fig. 1).

The patient was afebrile and presented no specific complaints.

The diagnosis of PUBS was made with the help of the Oxford urine chart. A dipstick urinalysis as well as a cytobacteriological examination of the urine $(\mathrm{CBEU})$, collected via the indwelling bladder catheter, was carried out and revealed alkaline urine $(\mathrm{pH}=8)$ with microscopic haematuria and leukocyturia without nitrites. The bladder catheter was changed and the patient received empiric antibiotic therapy with Levofloxacin $500 \mathrm{mg}$ once daily. The urine culture was subsequently positive for Proteus mirabilis (growth $>100,000 \mathrm{CFU} / \mathrm{mL}$ ), which is sensitive to quinolones, beta-lactams and nitrofurantoin. After obtaining the antibiogram, the targeted antibiotic therapy was adapted with oral Cefuroxime $500 \mathrm{mg}$ three times a day for a total duration of seven days of antibiotic therapy.

No recurrence of purple urine was observed.

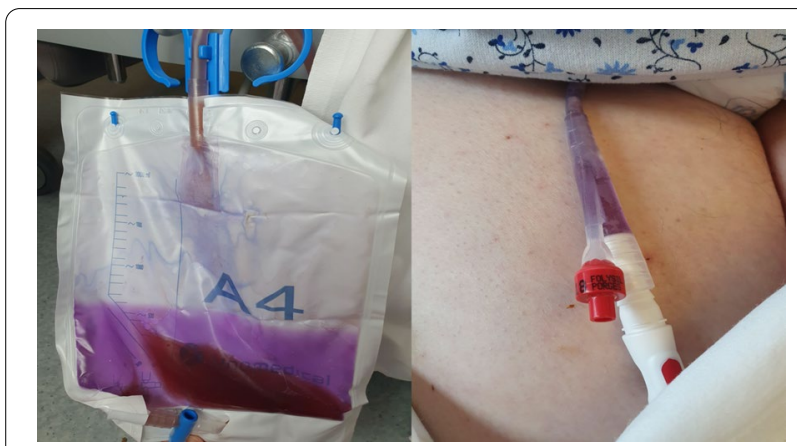

Fig. 1 Purple urine in the catheter bag as well as the tubing

\section{Discussion}

PUBS was first reported in 1978 in The Lancet [2].

Although this manifestation of bacteriuria is rare and poorly reported, its prevalence in certain cohort studies of patients with long-term bladder catheterisation has been estimated at $9.8 \%$ in chronic care units and up to $42.1 \%$ in nursing and care homes $[3,4]$.

The physiopathological hypothesis (Fig. 2) held by the authors is mainly based on the fact that the purple urine is a mixture of indigo and indirubin, which are derivatives of the metabolites of tryptophan. Ingested tryptophan is deaminated by the digestive bacterial flora to produce indole. The latter is transported in the portal circulation to the liver, where it is conjugated with indoxyl sulphate. Indoxyl sulphate is then secreted in the urine where it is transformed into indoxyl by the sulphatases and phosphatases produced by certain bacteria. Particularly in alkaline urine, indoxyl is oxidised to indirubin (red in colour) and indigo (blue in colour). These two pigments mix and react with the plastic of the tubing and the urine bag, which contain polyvinyl chloride (PVC), leading to the production of this purple tint in the urine. Many bacteria are associated with the occurrence of PUBS, mostly gram-negative bacilli such as Escherichia coli, Enterococcus species, Klebsiella pneumoniae, Providencia stuartii, Providencia rettgeri, Morganella morganii, Pseudomonas aeruginosa and Proteus mirabilis [5, 6]. It has nevertheless been established that not all bacteria of the same species produce sulphatases or phosphatases $[5,7,8]$.

Many risk factors are associated with the occurrence of PUBS, such as female sex, a diet rich in tryptophan, constipation, chronic bladder catheterisation, use of PVC plastic bladder catheters, alkaline urinary $\mathrm{pH}$, high urinary bacterial load and renal failure [8].

Concerning the reported case, severe constipation and rectal faecal impaction probably contributed to the genesis of PUBS. This likely occurred, on the one hand, by inducing acute retention of urine which may have favoured the proliferation of Proteus mirabilis in the urine, and, on the other hand, by promoting digestive bacterial proliferation which may have increased the deamination of tryptophan, thus increasing the substrate. The female genitourinary system is anatomically disposed to UTIs. The use of a PVC plastic bladder catheter, the alkaline urinary $\mathrm{pH}$ as well as the presence of a high urinary bacterial load of Proteus mirabilis are other factors that contributed to the development of PUBS in our patient. According to the patient's daughter, the patient did not benefit from a diet rich in tryptophan (dried dates, milk, yogurt, cottage cheese, red meat, eggs, fish, etc.) and only takes risperidone once daily, meaning that these are not responsible for the changes in the colour of the urine in this case. It is interesting to note that the 


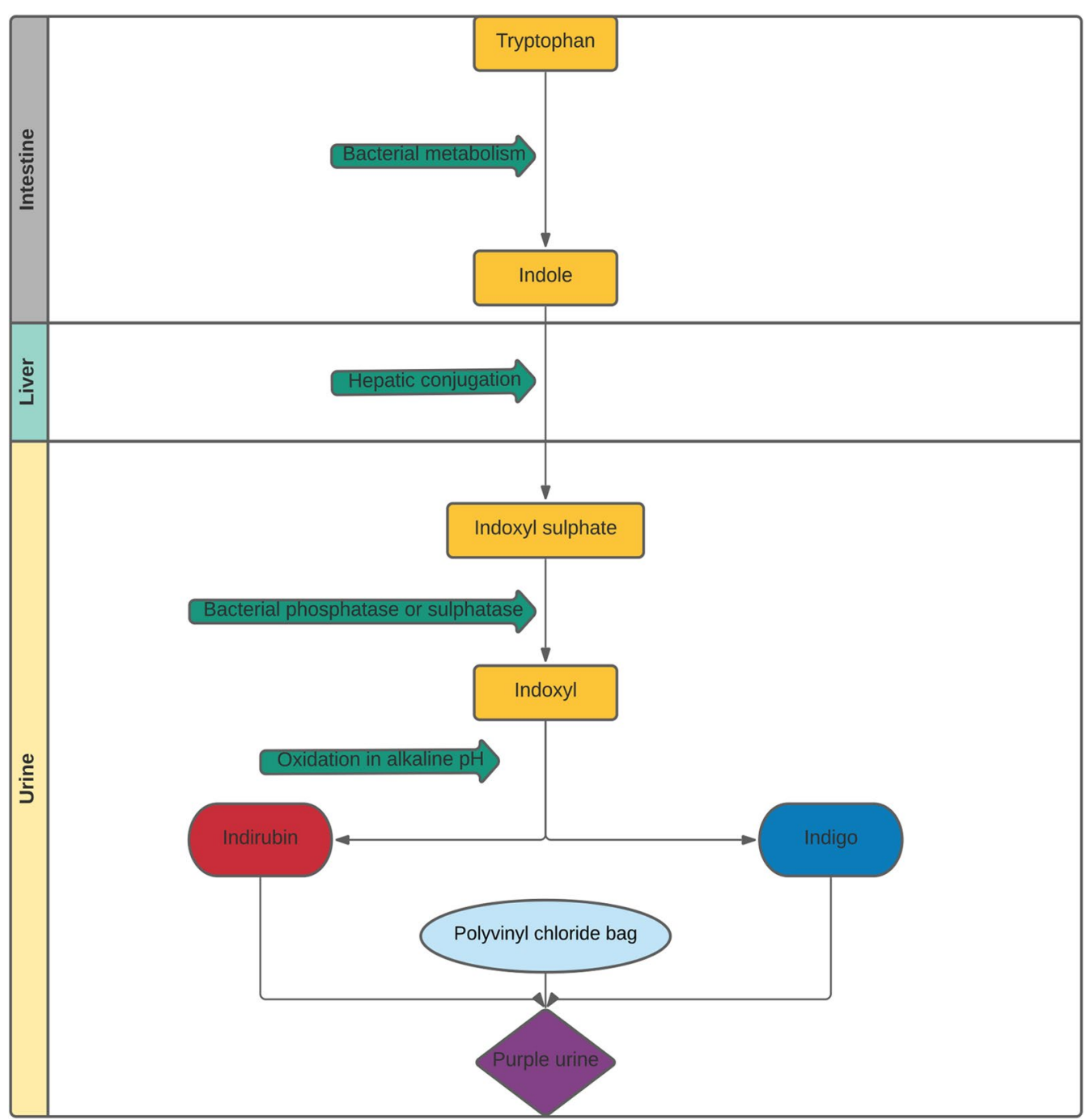

Fig. 2 Physiopathological hypothesis of PUBS

appearance of PUBS probably requires the simultaneous presence of several factors, and although alkaline urinary $\mathrm{pH}$ appears to be a key component in the process of purple urine formation, a case of PUBS with acidic urine has already been reported [9].

There are many aetiologies that can induce changes in the colour of the urine, such as certain diets (e.g. blackberries, beets, etc.), drugs (e.g. rifampicin, propofol, nitrofurantoin, etc.), hemoglobinuria, hematuria or porphyria. Therefore, there is undoubtedly a risk of erroneous diagnosis and, consequently, inappropriate treatment. It seems advisable that an Oxford urine chart has been developed to help the medical or nursing team to orient themselves towards a set of suspected aetiologies based on the colour of the urine presented by the patient [1]. In addition to the urine's colour, the presence of risk factors may also guide the diagnosis. We do not find any other cause of purple urine in the literature other than PUBS, so it is an easy clinical diagnosis. In case of suspicion of PUBS, a dipstick urinalysis as well as a CBEU must be carried out.

There is no evidence that the occurrence of PUBS as a urinary tract infection (UTI) has a prognostic impact in patients who develop this rare complication. However, it seems that PUBS is grafted with an increased relative morbidity based on rare cases described in the literature. Indeed, it has been noted to have complicated into a vulvar abscess with multi-resistance to antibiotics [10] or preceded the appearance of Fournier's gangrene [11].

To date, there are no guidelines governing the management of PUBS. While the attitude of changing the bladder catheter seems to be unanimous, the initiation of 
antibiotic treatment remains contested and must be discussed on a case-by-case basis [12]. The indications for antibiotic therapy could include symptomatic UTI, sepsis, immunosuppression or the extension of the infection by contiguity to neighbouring tissues $[1,13,14]$. The benefit of antibiotic therapy in patients in palliative care remains to be established, especially as antibiotic therapy in these patients can cause discomfort through side effects [15].

We decided to treat our patient with antibiotic therapy because she presented a biological inflammatory syndrome and could not express any urinary complaints given her decompensated psychiatric pathology.

\section{Conclusion}

PUBS is a rare complication of bacteriuria, which induces a purple colouration of the tubing as well as the catheter bag. It is a simple spot diagnosis, as there is no other known cause of purple urine. It is for this reason that we believe that the Oxford urine chart represents a very interesting and easily accessible tool to help clinicians to investigate any abnormal urine colour. Given the lack of clear recommendations for the management of PUBS, it seems reasonable to change the bladder catheter and to initiate antibiotherapy on a case-by-case basis, as was the case for our patient.

\section{Abbreviations}

PUBS: Purple urine bag syndrome; CBEU: Cytobacteriological examination of urine; PVC: Polyvinyl chloride; UTI: Urinary tract infection.

\section{Acknowledgements}

Not applicable.

\section{Authors' contributions}

$\mathrm{OM}$ : Took care of the patient during the hospitalization and wrote the article. TR: Took care of the patient during the hospitalization and helped write the article and proofread. MF, FL and CR : Helped write the article and proofread. All authors have read and approved the manuscript.

\section{Funding}

No funding needed for this study.

\section{Availability of data and materials}

The datasets used/or analyzed during the current study are available from the corresponding author on reasonable request.

\section{Declarations}

Ethics approval and consent to participate Not applicable.

\section{Consent for publication}

A written informed consent was obtained from the family of the patient for publication of this case report. The patient is unable to give consent (due to the psychiatric disorder).

\section{Competing interests}

The authors declare that they have no competing interests.

Received: 31 March 2021 Accepted: 12 June 2021

Published online: 24 June 2021

References

1. Kalsi DS, Ward J, Lee $R$ et al (2017) Purple urine bag syndrome: a rare spot diagnosis. Dis Markers 2017:9131872

2. Barlow GB, Dickson JAS (1978) Purple urine bags. Lancet 311:220-221

3. Dealler SF, Belfield PW, Bedford M et al (1989) Purple urine bags. J Urol 142:769-770

4. Lin CH, Huang HT, Chien CC et al (2008) Purple urine bag syndrome in nursing homes: ten elderly case reports and a literature review. Clin Interv Aging 3(4):729-734

5. Dealler SF, Hawkey PM, Millar MR (1988) Enzymatic degradation of urinary indoxyl sulfate by Providencia stuartii and Klebsiella pneumoniae causes the purple urine bag syndrome. J Clin Microbiol 26:2152-2156

6. Ollapallil J, Irukulla S, Gunawardena I (2002) Purple urine bag syndrome. ANZ J Surg 72:309-310

7. Jones RA, Deacon HJ, Allen SC (2003) Two cases and a short discussion of purple urine bag syndrome. CME Geriatr Med 5(2):84-87

8. Khan F, Chaudhry MA, Qureshi N et al (2011) Purple urine bag syndrome: an alarming hue? A brief review of the literature. Int J Nephrol 2011:419213

9. Chung SD, Liao CH, Sun HD (2008) Purple urine bag syndrome with acidic urine. Int J Infect Dis 12:526-527

10. Dharmshaktu GS, Pangtey T (2018) Purple urine bag as indicator of multidrug resistant vulvar abscess: lessons for primary caregivers. J Fam Med Primary Care 7(2):484-485

11. Tasi YM, Huang MS, Yang CJ (2009) Purple urine bag syndrome not always a benign process. Am J Emerg Med 27(7):895-897

12. Bhattarai M, Bin Mukhtar H, Davis TW, Silodia A, Nepal H (2013) Purple urine bag syndrome may not be benign: a case report and brief review of the literature. Case Rep Infect Dis 2013:3

13. Hadano Y, Shimizu T, Takada S et al (2012) An update on purple urine bag syndrome. Int J Gen Med 5:707-710

14. Su FH, Chung SY, Chen MH et al (2005) Case analysis of purple urine-bag syndrome at a long-term care service in a community hospital. Chang Gung Med J 28(9):636-642

15. Wattanapisit S, Wattanapisit A, Meepuakmak A et al (2019) Purple urine bag syndrome in palliative care. BMJ Support Palliat Care 9:155-157

\section{Publisher's Note}

Springer Nature remains neutral with regard to jurisdictional claims in published maps and institutional affiliations. 